OPEN ACCESS

Edited by:

Weifeng Zhao,

Sichuan University,

China

Reviewed by:

Yang Kang

Chengdu Institute of Biology (CAS)

China

Zhipeng Gu,

Sun Yat-sen University, China

*Correspondence:

Shan-hui Hsu

shhsu@ntu.edu.tw

Specialty section:

This article was submitted to

Polymer Chemistry,

a section of the journal

Frontiers in Chemistry

Received: 03 August 2018 Accepted: 07 September 2018

Published: 02 October 2018

Citation:

Liu Y and Hsu S (2018) Synthesis and

Biomedical Applications of

Self-healing Hydrogels.

Front. Chem. 6:449.

doi: 10.3389/fchem.2018.00449

\section{Synthesis and Biomedical Applications of Self-healing Hydrogels}

\author{
Yi Liu ${ }^{1}$ and Shan-hui Hsu ${ }^{1,2 *}$ \\ ${ }^{1}$ Institute of Polymer Science and Engineering, National Taiwan University, Taipei, Taiwan, ${ }^{2}$ Institute of Cellular and System \\ Medicine, National Health Research Institutes, Miaoli, Taiwan
}

Hydrogels, which are crosslinked polymer networks with high water contents and rheological solid-like properties, are attractive materials for biomedical applications. Self-healing hydrogels are particularly interesting because of their abilities to repair the structural damages and recover the original functions, similar to the healing of organism tissues. In addition, self-healing hydrogels with shear-thinning properties can be potentially used as the vehicles for drug/cell delivery or the bioinks for 3D printing by reversible sol-gel transitions. Therefore, self-healing hydrogels as biomedical materials have received a rapidly growing attention in recent years. In this paper, synthesis methods and repair mechanisms of self-healing hydrogels are reviewed. The biomedical applications of self-healing hydrogels are also described, with a focus on the potential therapeutic applications verified through in vivo experiments. The trends indicate that self-healing hydrogels with automatically reversible crosslinks may be further designed and developed for more advanced biomedical applications in the future.

Keywords: self-healing hydrogel, synthesis mechanism, reversible crosslink, biomedical application, animal model

\section{INTRODUCTION}

Hydrogels are constructed by the crosslinked polymer networks as water-swollen gels. Hydrogels have received significant attention as the extracellular matrix mimics for biomedical applications because of their water-retention abilities, appropriate elasticities, and network structures (Wang and Heilshorn, 2015). The self-healing properties, originated from phenomena of wound healing in organisms, are used to describe materials with the ability to restore the morphology and mechanical properties after repeated damages. The microcapsule-laden hydrogels were developed that released healing agents at damage sites (White et al., 2001; Toohey et al., 2007). However, the irreversible healing process and potential interference of fillers limited their applications (Bergman and Wudl, 2008; Syrett et al., 2010). Besides, many dynamic hydrogels typically relied on external stimuli, such as high temperature, low $\mathrm{pH}$, and light, to trigger dynamic crosslinks (Murphy and Wudl, 2010; Harada et al., 2014). The external stimuli would have adverse effects on the cells and living tissues. In this review, self-healing hydrogels are referred that automatically and reversibly repair the damages and recover the functions.

Self-healing hydrogels can be prepared through dynamic covalent bonds and non-covalent interactions. The dynamic equilibrium between dissociation and recombination of various interactions leads the hydrogel to heal damages and reform shapes. Commonly, dynamic covalent bonds exhibit stable and slow dynamic equilibriums, while non-covalent interactions show fragile and rapid dynamic equilibriums (Zou et al., 2017). With versatile mechanical properties, 
self-healing hydrogels can be manufactured with robust, shearthinning, or cell-adaptable properties for a broad range of applications, such as soft robots, 3D printing, and drug/cell delivery. In this review paper, we will take a detailed look at the current synthesis and biomedical applications of self-healing hydrogels. Firstly, various advanced strategies are introduced about the preparations and the mechanisms of self-healing hydrogels. Subsequently, biomedical applications of the selfhealing hydrogels are described, especially, the ones that have been evaluated by animal models.

\section{SELF-HEALING MECHANISMS}

Self-healing hydrogels have been synthesized based on different chemistries and mechanisms as shown in Figure 1, including dynamic covalent bonds, non-covalent interactions, and multimechanism interactions. Each will be elaborated below.

\section{Dynamic Covalent Bonding}

Dynamic covalent chemistry, including imine formation, boronate ester complexation, catechol-iron coordination, DielsAlder reaction, and disulfide exchange, is widely applied in the formation of self-healing hydrogel. Dynamic covalent bonds exhibit the stronger but slower dynamic equilibrium compared to non-covalent interactions.

The imine (or referred as Schiff base) is a compound with a carbon-nitrogen double bond formed by nucleophilic attack of amine to aldehyde or ketone. A number of self-healing hydrogels have been developed by aliphatic Schiff bases (Lü et al., 2015; Zhu, D. et al., 2017; Huang et al., 2018) or aromatic Schiff bases (Karimi and Khodadadi, 2016; Qu et al., 2017), in which aromatic Schiff bases show higher stability to maintain the mechanical properties compared to aliphatic Schiff bases (Zhang et al., 2011). Zhang et al. synthesized a dibenzaldehyde-terminated telechelic poly(ethylene glycol), namely difunctionalized PEG (DF-PEG), to form self-healing hydrogel through aromatic Schiff bases between benzaldehyde groups of DF-PEG and amino groups of chitosan (Zhang et al., 2011). The hydrogels were prepared rapidly under mild conditions at $20^{\circ} \mathrm{C}$ within $60 \mathrm{~s}$, and they could be degraded by acidic $\mathrm{pH}$, amino acids, vitamin $\mathrm{B} 6$ derivatives, and enzymes. The hydrogels were developed for $3 \mathrm{D}$ cell culture and cell delivery due to their cytocompatibility and injectability (Yang et al., 2012; Li et al., 2017; Zhang, Y. L. et al., 2017). Acylhydrazone and oxime are derivatives of imine with great stability, which have also been developed to synthesize the selfhealing hydrogels (Deng et al., 2010; Grover et al., 2012; Lin et al., 2013; Mukherjee et al., 2015). For example, the self-healing hydrogel was prepared by adding oxidized sodium alginate into the mixture of $\mathrm{N}$-carboxyethyl chitosan and adipic acid dihydrazide via dynamic imine and acylhydrazone bonds (Wei et al., 2015).

The reversible boronate ester bond is formed by complexation of boronic acid and diol, and its stability is dependent on $\mathrm{pH}$-value and glucose concentration. Boronic acid and its derivatives, such as phenylboronic acid or phenylboronic acidincorporated polymers, have been widely developed to prepare self-healing hydrogels. Yesilyurt et al. mixed phenylboronic acid and diol-modified poly(ethylene glycol) to form self-healing hydrogel that exhibited $\mathrm{pH}$-responsive tunable mechanical properties, and glucose-responsive size-dependent release of proteins (Yesilyurt et al., 2016). The hydrogel was cytocompatible in vitro, and it showed a typical foreign body reaction in vivo without chronic inflammation. He et al. prepared selfhealing hydrogel via complexation of a catechol-modified polymer and 1,3-benzenediboronic acid, which demonstrated high stability under alkaline conditions and low stability under acidic conditions (He et al., 2011). Another self-healing hydrogel was fabricated using the mixture of poly(ethylene glycol) diacrylate, dithiothreitol, and borax via permanent thiol-ene Michael addition and dynamic borax diol complexation in onepot approach (He et al., 2015). The hydrogel can be injected with cells to form branched tubular channels for vascularization in vitro and easily removed by immersion in cell culture medium (Tseng et al., 2017).

The reversible coordinate bond between catechol and iron has been developed to prepare self-healing hydrogels. The reversibility of catechol-iron coordination bond can be controlled by adjusting pH conditions (Krogsgaard et al., 2013). When the environmental $\mathrm{pH}$ is raised from acidic to basic values, a rapidly self-healing hydrogel with high strength may form. $\mathrm{Li}$ et al. incorporated iron oxide $\left(\mathrm{Fe}_{3} \mathrm{O}_{4}\right)$ nanoparticles with the catechol-modified polymers to form a self-healing hydrogel via reversible coordination bonds at the nanoparticle surface (Li et al., 2016). Self-healing hydrogel based on catechol- $\mathrm{Fe}_{3} \mathrm{O}_{4}$ nanoparticles structures exhibited magnetic properties and solidlike mechanics, in comparison with the fluid-like hydrogel by catechol-Fe(III) crosslinking.

The disulfide exchange provides dynamic covalent bonds to form self-healing hydrogels, which are sensitive to $\mathrm{pH}$ or redox potential (Wei, Z. et al., 2014). Recently, 1,2-dithiolanefunctionalized polymers were synthesized to form self-healing hydrogels with rapid sol-gel transition via the disulfide exchange between the 1,2-dithiolane and dithiols (Barcan et al., 2015; Yu et al., 2017; Zhang and Waymouth, 2017). The disulfide exchange of 1,2-dithiolane can reform under neutral or weakly alkaline conditions, which can be further controlled by temperature.

Another important dynamic covalent chemistry in selfhealing hydrogels is the thermally reversible Diels-Alder reaction (Liu and Chuo, 2013; Zhao et al., 2016; Shao et al., 2017). However, the biomedical applications of Diels-Alder reaction are limited because Diels-Alder bonds need a high temperature and a long duration to cleave and reform for self-healing properties. In recent reports, Diels-Alder chemistry was developed to form selfhealing hydrogels combining with other reversible interactions, such as electrostatic interaction (Banerjee and Singha, 2017; Ghanian et al., 2018), coordination bond (Li et al., 2018a), imine bond (Li et al., 2018b), and acylhydrazone bond (Yu et al., 2015).

\section{Non-covalent Interactions}

Self-healing hydrogels can be produced through non-covalent interactions, such as hydrogen bond, electrostatic interaction, and hydrophobic interaction. The non-covalent interactions are less stable and more sensitive to environmental conditions (such as $\mathrm{pH}$ and temperature) compared to covalent interactions. 


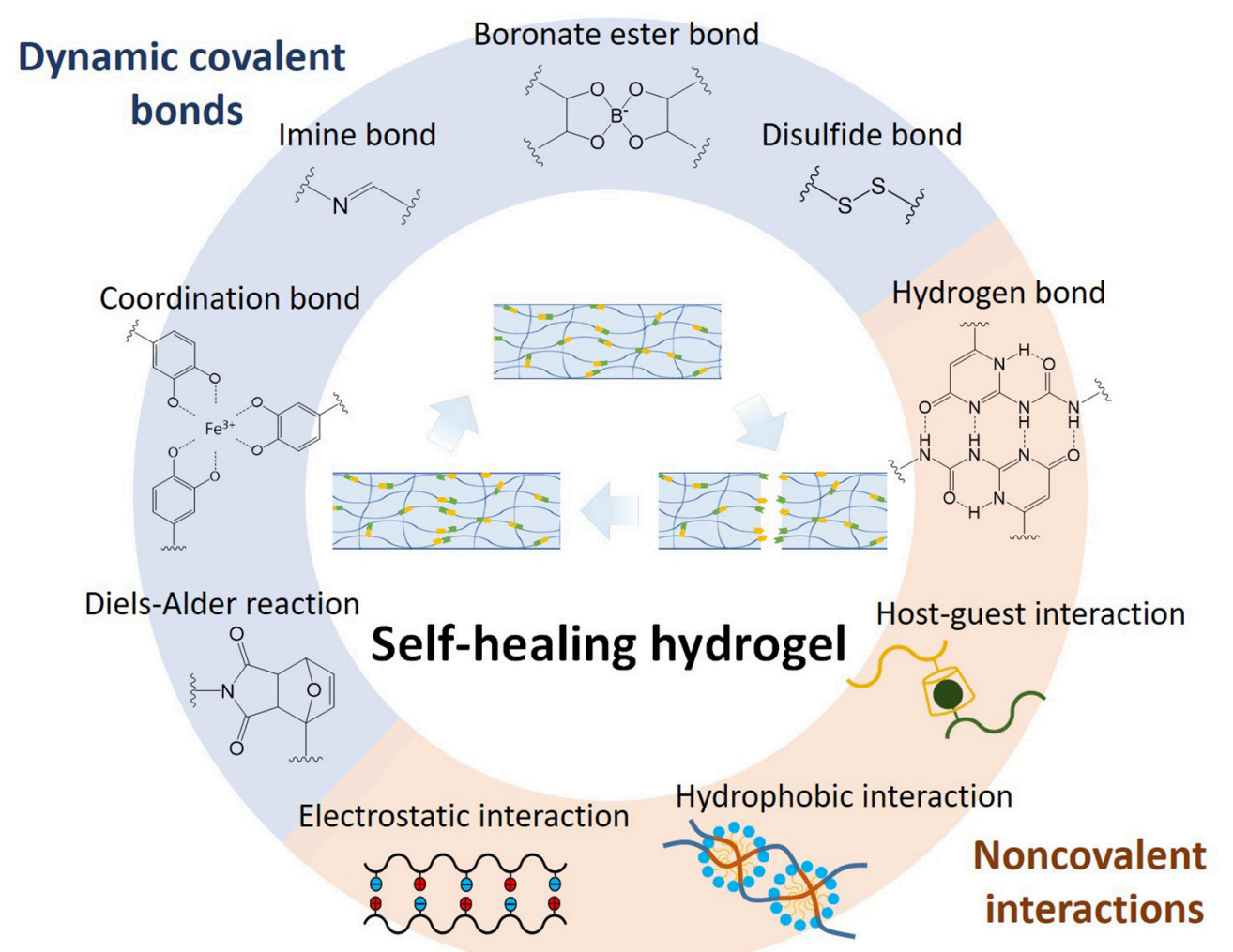

FIGURE 1 | Self-healing chemistries and mechanisms for various self-healing hydrogels, including dynamic covalent bonds, non-covalent interactions, and multi-mechanism interactions.

However, robust self-healing hydrogels can still form based on non-covalent interactions via special manufacturing procedures or nano- and micro-structures.

The hydrogen bonding is an attractive interaction between the hydrogen atoms and electronegative atoms, in which the hydrogen atom is bound to a high electronegative atom, such as nitrogen, oxygen, and fluorine. The polyvinyl alcohol-based self-healing hydrogels were developed using the freezing/thawing method via hydrogen bonding (Zhang et al., 2012; Zhang, Z. et al., 2017). Moreover, hydrogen bonding-based self-healing hydrogels were frequently reported with incorporations of various chemical moieties, such as 2-ureido-4-pyrimidone (UPy) moieties (Cui and del Campo, 2012; Dankers et al., 2012; Cui et al., 2013; Bastings et al., 2014; Chirila et al., 2014; Hou et al., 2015; Zhang et al., 2016), nucleobase moieties (Ye et al., 2017), deferoxamine moieties (Xu et al., 2017), and gallol moieties (Shin and Lee, 2017). In a recent work, the cytosine- and guanosine-modified hyaluronic acid (HA) formed self-healing hydrogel by Watson-Crick base pairing between the nucleobases through hydrogen bonding (Ye et al., 2017). The hydrogel exhibited $\mathrm{pH}$-stimulated sol-gel transition where the hydrogel exhibited gel state in $\mathrm{pH} 6-8$ and sol state in $\mathrm{pH}<6$ or $>8$. In another example, Shin and Lee synthesized gallol-conjugated $\mathrm{HA}$ and added a gallolrich crosslinker (i.e., oligo-epigallocatechin gallate) to form a shear-thinning and self-healing hydrogel based on extensive hydrogen bonds of gallol-gallol and gallol-HA (Shin and Lee, 2017). The hydrogel was resistant to enzymatic degradation by protein (i.e., hyaluronidase) immobilization through noncovalent interactions between gallols and proteins.

Hydrophobic interactions occur as a consequence of aggregative hydrophobes in aqueous media. In many cases of self-healing hydrogels based on hydrophobic interactions, the surfactant micelles (Gulyuz and Okay, 2015; Liu, Y. et al., 2018) or liposomes (Rao et al., 2011; Hao et al., 2013) are employed as crosslinking points to construct the polymer chains comprising both hydrophilic and hydrophobic monomers. For example, the self-healing hydrogel could form via micellar copolymerization of hydrophobic monomer stearyl methacrylate and hydrophilic monomer acrylamide in the aqueous solution of sodium dodecyl sulfate (SDS) micelles (Tuncaboylu et al., 2011, 2012a,b). In these cases, the addition of salt into aqueous SDS solutions leads to micellar growth and solubilization of hydrophobes within SDS micelles. The hydrogel containing SDS micelles with the time-dependent dynamic moduli exhibited high elongation ratio and good self-healing ability, while after extraction of SDS, the hydrogel with time-independent dynamic moduli showed high mechanical strength and no self-healing ability. Self-healing hydrogel can also be prepared based on surfactantfree hydrophobic associations via solvent evaporation of an 
aqueous polymer solution above a critical polymer concentration (Owusu-Nkwantabisah et al., 2017).

Self-healing hydrogels can form through reversible electrostatic interactions occurring in charged polymers and ions (Wei et al., 2013; Wei, H. et al., 2014), polyelectrolytes (Huang et al., 2014; Luo et al., 2015; Ren et al., 2016; Li, J. et al., 2017), polyampholytes (Ihsan et al., 2013; Sun et al., 2013), and zwitterionic fusions (Bai et al., 2014). For example, self-healing hydrogel was synthesized through reversible polyelectrolyte complexes of alginate and 2-hydroxypropyltrimethyl ammonium chloride chitosan (Ren et al., 2016). The two polymers were mixed to form self-healing hydrogel at charge neutrality followed by precipitation for $12 \mathrm{~h}$. In addition to self-healing ability, the hydrogel exhibited shear-thinning property, high adhesive behavior, and cytocompatibility. Meanwhile, the polyampholytes can form self-healing hydrogels with tunable mechanical properties via electrostatic interactions between randomly dispersed cationic and anionic repeating groups in polymers (Sun et al., 2013). In analogy to double-network hydrogels, the tough polyampholyte hydrogels contained ionic strong bonds and weak bonds to maintain the shapes and enhance the shock absorbance and self-healing abilities, respectively. Besides, the more hydrophobic polyampholyte hydrogels exhibited the robust and poor self-healing properties, whereas the less hydrophobic polyampholyte hydrogels exhibited the soft and good self-healing properties (Sun et al., 2013).

\section{Multi-mechanism Interactions}

Supramolecular chemistry is widely applied to prepare selfhealing hydrogels through various non-covalent interactions, such as host-guest interaction and protein-ligand recognition. In addition, hybrids of non-covalent interactions and/or permanent/dynamic covalent bonds were developed to prepare self-healing hydrogels for rapid recovery, long-term stability, high mechanical property, and/or multi-responsive behavior.

Host-guest interactions occur when two or more chemical species assemble via non-covalent interactions, such as van der Waals force, hydrogen bond, electrostatic interaction, and hydrophobic interaction. In host-guest chemistry, the macrocyclic host moiety is inserted inside the guest moiety to form a unique structure of the inclusion complexation. Hostguest interactions are used popularly to prepare the self-healing hydrogels, and many such hydrogels rely on external stimuli, such as temperature (Zheng et al., 2012), light (Yamaguchi et al., 2012), pH (Zheng et al., 2013), and redox potentials (Nakahata et al., 2011; Miyamae et al., 2015), to trigger the healing process. Meanwhile, host-guest hydrogels have also been developed to recover themselves without the need of external stimuli (Appel et al., 2012; Kakuta et al., 2013; Rodell et al., 2013; McKee et al., 2014). For example, the self-healing HA hydrogel was prepared based on the host-guest interactions of $\beta$-cyclodextrinmodified HA (host macromer) and adamantane-modified HA (guest macromer) (Rodell et al., 2013). The hydrogels exhibited shear-thinning property and rapid recovery at $25^{\circ} \mathrm{C}$.

Catechol and gallol are polyphenolic moieties commonly distributed in organisms as important functional groups, which can form various covalent and non-covalent bonds, such as Michael addition or Schiff base reaction with thiol and amine, coordination bonds with metals, hydrogen bonds, and aromatic interactions (Lee et al., 2007; Sileika et al., 2013). Li et al. developed a novel self-healing hydrogel by selfassembly of an ABA tri-block copolymer through the catecholmediated hydrogen bonding and aromatic interaction, where the catechol-functionalized poly(N-isopropylacrylamide) (PNIPAM) and poly(ethylene oxide) (PEO) were each selected as A and B blocks for synthesis (Li et al., 2015). The hydrogel exhibited a thermo-responsive sol-gel transition and recovered its mechanical properties after repeated damages owing to PNIPAM moiety and catechol-mediated interaction, respectively. Moreover, Birkedal and coworkers prepared a self-healing and $\mathrm{pH}$-responsive hydrogel using tannic acid (TA), metal ions, and polyallylamine (PAA) in one step (Krogsgaard et al., 2014a). Below pH 8, the hydrogel was crosslinked mostly by reversible hydrogen bonds, covalent crosslinks between TA and PAA, and coordination bonds between TA and iron ion; while above $\mathrm{pH} 8$, irreversible bonds predominantly enhanced the gel modulus and hindered self-healing. Likewise, selfhealing hydrogels were developed based on interactions between 3,4-dihydroxyphenylalanine-modified PAA (DOPA-PAA) and metal ions [such as $\mathrm{Al}(\mathrm{III}), \mathrm{Ga}(\mathrm{III}), \mathrm{In}(\mathrm{III})$, and $\mathrm{Fe}(\mathrm{III})$ ions; (Krogsgaard et al., 2014b)].

Dupin and coworkers reported for the first time the formation of self-healing hydrogels using gold(I) ions-crosslinked thiolterminated PEG via metallophilic attractive forces (Casuso et al., 2014). The hydrogel exhibited cytocompatibility and mimicked the synovial fluid of the human joint in rheological properties under physiological conditions. Afterward, a series of self-healing hydrogels with tunable mechanical properties were prepared using $\mathrm{HAuCl}_{4}$ (or $\mathrm{AgNO}_{3}$ ) and 4-arm thiolterminated polyethylene glycol $\left[(\mathrm{PEGSH})_{4}\right]$ in different ratios based on metal(I)-thiolate/disulfide exchange (Casuso et al., 2015). These hydrogels showed reversible mechanical properties and frequency-dependent stiffness/shock-absorbing properties at the physiological $\mathrm{pH}$ due to the metal(I)-thiolate/disulfide exchange. The potential of the hydrogel as an artificial nucleus pulposus for the intervertebral discs was demonstrated via a bovine ex vivo model using axial compression-tension cycles at different frequencies followed by creep experiments and $\mu \mathrm{CT}$ analysis (Pérez-San Vicente et al., 2017). Moreover, the hydrogels incorporating bioactive glass nanoparticles led to the stiffer properties for bone regeneration (Gantar et al., 2016). Meanwhile hydroxyapatite was formed after degradation of the nanoparticles.

On the basis of dynamic acylhydrazone and disulfide bonds, self-healing hydrogels with $\mathrm{pH} /$ redox dual responsive transitions have been developed (Deng et al., 2012). The hydrogel displayed self-healing properties in acidic and basic conditions based on the acylhydrazone and disulfide bonds, respectively. Additionally, acylhydrazone bonds were activated by the catalytic aniline in neutral conditions, and disulfide bonds were responsive to the redox conditions. Recently, the self-healing hydrogel was prepared from the mixture carboxyethyl cellulose-graftdithiodipropionate dihydrazide and DF-PEG under 4-aminoDL-phenylalanine (4a-Phe) catalysis (Yang et al., 2017). The 
gelation time of the hydrogel could be controlled by varying the total polymer content or the $4 \mathrm{a}$-Phe concentration. The hydrogel was applied for controlled release of doxorubicin and $3 \mathrm{D}$ culture of L929 cells because of $\mathrm{pH} /$ redox responsiveness and cytocompatibility.

\section{BIOMEDICAL APPLICATIONS OF SELF-HEALING HYDROGELS}

Self-healing hydrogels have received increasing attentions in biomedical applications, such as wound healing (Gaharwar et al., 2014; Han et al., 2016; Zhao et al., 2017; Zhu, S. K. et al., 2017; Li et al., 2018; Liu, B. et al., 2018), drug delivery (Huebsch et al., 2014; Liu et al., 2016; Wang et al., 2016; Xing et al., 2016; Wang J. Y. et al., 2017; Xia et al., 2017; Yavvari et al., 2017; Zhu, C. et al., 2017; Hong et al., 2018), tissue engineering (Dankers et al., 2012; Bastings et al., 2014; Gaffey et al., 2015; Rodell et al., 2015a,b; Loebel et al., 2017), surface coating (Canadell et al., 2011; Yoon et al., 2011; Yang et al., 2015), 3D printing (Highley et al., 2015; Darabi et al., 2017; Loebel et al., 2017; Wang et al., 2018), and soft robot (Shi et al., 2015; Darabi et al., 2017; Han et al., 2017; Liu, B. et al., 2018; Liu et al., 2018). In these cases, dibenzaldehyde-based, UPy-based, catechol-based, and host-guest-based self-healing hydrogels are highlighted due to many evaluations of in vivo experiments. As summarized in Table 1, some animal models have been used to verify the biocompatibility and efficacy of self-healing hydrogels. Besides the biocompatibility, self-healing hydrogels require injectability and long-term stability for drug delivery, tissue engineering, and 3D printing; and toughness and conductivity for soft robot.

\section{Drug Delivery}

Self-healing hydrogel based on host-guest interactions between $\beta$-cyclodextrin-modified PEI and adamantane-modified PEG was developed for local siRNA release (Wang et al., 2018). The modified polymers assembled with siRNA to form polyplexes, which could improve the transfection efficiency and the viability of cells. When injected into the myocardium, the hydrogel with siRNA encapsulation enhanced the uptake of Cy5.5-siRNA and maintained the silencing of GFP for 1 week in a GFP-expressing rat.

Xing et al. reported an injectable and self-healing collagengold hybrid hydrogel with adjustable mechanical properties (Xing et al., 2016). This hydrogel was prepared through electrostatic interaction between positively charged collagen chains and negatively charged tetrachloroaurate $\left(\left[\mathrm{AuCl}_{4}\right]^{-}\right)$ ions, and further non-covalent interactions between subsequent biomineralized gold nanoparticles and collagen. The hydrogel was developed for localized delivery and sustained release of the photosensitive drug. By combinatorial photothermal and photodynamic therapies, the significantly enhanced antitumor efficacy was demonstrated through an in vivo antitumor test using the subcutaneous mouse model.

Self-healing hydrogels based on glycol chitosan and DFPEG (GC-DP) have been developed for intratumor therapy in vivo. GC-DP hydrogel containing antitumor drug was injected into the disease position with a steady release in situ (Yang et al., 2017). Moreover, the ionic GC-DP hydrogel exhibited microwave susceptibility to produce high-temperature hyperthermia for tumor ablation (Wang J. Y. et al., 2017). A multi-antitumor system was developed based on GC-DP hydrogel containing doxorubicin/docetaxel-loaded poly(lacticco-glycolic acid) (PLGA) nanoparticles and iron oxide for chemotherapy and magnetic hyperthermia (Xie et al., 2017). The system showed the greater in vivo antitumor efficacy under the alternative magnetic field compared to the hydrogel containing doxorubicin/docetaxel-loaded PLGA nanoparticles.

\section{Tissue Engineering}

Self-healing host-guest hydrogels have been developed to treat the myocardial infarction. The self-healing hydrogel, formed through host-guest interactions of adamantine- and $\beta$-cyclodextrin-modified HA, was injected into the ischemic myocardium encapsulating endothelial progenitor cells (EPCs) (Gaffey et al., 2015). A rodent model of acute myocardial infarction was employed to confirm that a significant increase in vasculogenesis was noted with the hydrogel encapsulating EPCs, compared to the treatment of EPCs alone or hydrogel alone. Moreover, the hydrogel was designed using adamantane/thiolmodified HA and cyclodextrin/methacrylate-modified HA through host-guest interaction and Michael addition (Rodell et al., 2015a). The reversible host-guest interaction and permanent Michael addition provided shear-thinning injection and high retention, respectively. Epicardial injection of the hydrogel in a rat myocardial infarction model showed significant improvement of the outcome compared to the untreated group and the hydrogel without Michael addition.

Self-healing hydrogels were designed as injectable carriers for growth factors using PEG end-functionalized with four-fold hydrogen-bonding ureidopyrimidinone (UPy) moieties. UPymodified PEG hydrogel incorporated with antifibrotic growth factor was delivered in a pocket introduced under the kidney capsule of rats (Dankers et al., 2012). The kidney capsule was loosened from the kidney to create a small pocket. After injection of growth factor-containing hydrogels, the number of myofibroblasts stayed the same to the contralateral (healthy) kidney, while significantly increased with the injection of saline or hydrogel alone. In another example, growth factors were delivered by UPy-modified PEG hydrogel to repair the infarcted myocardium (Bastings et al., 2014). This $\mathrm{pH}$-switchable hydrogel could be injected through the long and narrow lumen of the catheter mapping system, and rapidly formed a hydrogel in contact with tissue. The growth factor-containing hydrogel reduced scar collagen in a chronic myocardial infarction pig model.

Self-healing hydrogels based on GC-DP was prepared for tissue repairs. In the application of central nervous system (CNS) repair, neurosphere-like progenitors showed better proliferation and differentiation in GC-DP hydrogel, and injection of GCDP hydrogel combining neurospheres promoted functional recovery in a zebrafish CNS impaired model (Tseng et al., 2015). Moreover, the GC-DP hydrogel combining the optogenetic method was developed as a temporal-spatial approach to treat 
TABLE 1 | Examples of self-healing hydrogels evaluated by animal models.

\begin{tabular}{|c|c|c|c|}
\hline Self-healing mechanisms & Materials & Animal model evaluation & References \\
\hline Boronate ester bonds & Alginate-boronic acid & Oral administration for drug retention & Hong et al., 2018 \\
\hline Coordination bonds & Dexamethasone phosphate and Ca(II) & Subcutaneous injection for drug delivery & Liu et al., 2016 \\
\hline Coordination bonds & Chitosan-catechol and Fe(III) & Cancer model for drug delivery & Yavvari et al., 2017 \\
\hline Coordination bonds and electrostatic interactions & Collagen and gold & Cancer model for drug delivery & Xing et al., 2016 \\
\hline Electrostatic interactions & Silicate nanoplatelets and gelatin & Liver bleeding model for hemostasis & Gaharwar et al., 2014 \\
\hline Hydrogen bonds & Polyglutamic acid and lysine & Skin defect model for wound healing & Zhu, S. K. et al., 2017 \\
\hline Hydrogen bonds & Ureidopyrimidinone-PEG & Kidney implantation for tissue repair & Dankers et al., 2012 \\
\hline Hydrogen bonds & Ureidopyrimidinone-PEG & Myocardial infarction model for tissue repair & Bastings et al., 2014 \\
\hline Hydrogen bonds & Gelatin methacrylate and tannic acid & Gastric incision model for wound closure & Liu, B. et al., 2018 \\
\hline Hydrogen bonds and aromatic interactions & $\begin{array}{l}\text { Polydopamine nanoparticles and } \\
\text { poly(N-isopropylacrylamide) }\end{array}$ & Skin defect model for wound healing & Han et al., 2016 \\
\hline Hydrogen bonds and aromatic interactions & $\begin{array}{l}\text { Polydopamine, graphene oxide, and } \\
\text { polyacrylamide }\end{array}$ & Osteochondral defect model for tissue repair & Han et al., 2017 \\
\hline Host-guest interactions & $\begin{array}{l}\beta \text {-Cyclodextrin-PEl and } \\
\text { adamantane-PEG }\end{array}$ & Myocardium injection for drug delivery & $\begin{array}{l}\text { Wang L. L. et al., } \\
2017\end{array}$ \\
\hline Host-guest interactions & $\begin{array}{l}\text { Adamantane/thiol-HA and } \\
\text { cyclodextrin/methacrylate-HA }\end{array}$ & Myocardial infarction model for tissue repair & Rodell et al., 2015a \\
\hline Host-guest interactions & $\begin{array}{l}\text { Adamantane-HA and } \\
\beta \text {-cyclodextrin-HA }\end{array}$ & Myocardial infarction model for tissue repair & Gaffey et al., 2015 \\
\hline Host-guest interactions & $\begin{array}{l}\text { Adamantane-HA and } \\
\beta \text {-cyclodextrin-HA }\end{array}$ & Chronic kidney disease model for drug delivery & Rodell et al., 2015b \\
\hline Imine bond & DF-PEG and chitosan-aniline tetramer & Subcutaneous injection for cell retention & Dong et al., 2016 \\
\hline Imine bond & $\begin{array}{l}\text { Chondroitin sulfate-aldehyde and } \\
\mathrm{N} \text {-succinyl-chitosan }\end{array}$ & $\begin{array}{l}\text { Subcutaneous injection for material } \\
\text { degradation }\end{array}$ & Lü et al., 2015 \\
\hline Imine bond & $\begin{array}{l}\text { DF-PEG-co-poly(glycerol sebacate) } \\
\text { and chitosan-polyaniline }\end{array}$ & Skin defect model for wound healing & Zhao et al., 2017 \\
\hline Imine bond & $\begin{array}{l}\text { Aldehyde-xanthan and } \\
\text { carboxymethyl-chitosan }\end{array}$ & Abdominal wall defect model for tissue repair & Huang et al., 2018 \\
\hline Imine bond & DF-PEG and glycol chitosan & Cancer model for drug delivery & Xia et al., 2017 \\
\hline Imine bond & $\begin{array}{l}\text { DF-PEG-co-poly(glycerol sebacate) } \\
\text { and Chitosan-polyaniline }\end{array}$ & Liver bleeding model for hemostasis & Zhao et al., 2017 \\
\hline Imine bond & $\begin{array}{l}\text { DF-PEG, glycol chitosan, fibrinogen, } \\
\text { and thrombin }\end{array}$ & Hindlimb ischemia model for tissue repair & Hsieh et al., 2017 \\
\hline Imine bond & DF-PEG and glycol chitosan & Zebrafish neural injury model for tissue repair & Tseng et al., 2015 \\
\hline Imine bond & DF-PEG and glycol chitosan & Zebrafish neural injury model for drug delivery & Hsieh et al., 2018 \\
\hline Imine bond & $\begin{array}{l}\text { DF-PEG, glycol chitosan, fibrinogen, } \\
\text { and thrombin }\end{array}$ & Zebrafish embryos injection for angiogenesis & Hsieh et al., 2017 \\
\hline
\end{tabular}

neurodegenerative diseases (Hsieh et al., 2018). The hydrogel containing bacteriorhodopsin plasmid and neural stem cells was injected into CNS impaired zebrafish where the neural repair was observed, particularly under green light exposure. Besides, GC-DP hydrogel was also used to induce blood capillary formation. With the incorporation of fibrin gel, a composite hydrogel could form with an interpenetrating polymer network (i.e., double network) of GC-DP and fibrin (Hsieh et al., 2017). The hydrogel induced vascular endothelial cells to form capillarylike structures, and injection of the hydrogel alone promoted angiogenesis in zebrafish and rescued the blood circulation in ischemic hindlimbs of mice.

\section{Other Applications}

Self-healing hydrogels, based on the host-guest interaction of $\beta$-cyclodextrin- and adamantine-modified HA, were used in the $3 \mathrm{D}$ printing of high-resolution structures through printing of shearing-thinning ink hydrogel into self-healing support hydrogel (Highley et al., 2015). The multicellular structures could be expediently patterned, such as printing of mesenchymal stem cells within an ink hydrogel into a support hydrogel containing 3T3 fibroblasts. The channel-like structure was achieved by writing the ink hydrogel into the methacrylate-modified support hydrogel, followed by UV irradiation for secondary covalent crosslinks of support hydrogel, followed by removal of the physical (i.e., host-guest) ink hydrogel. Meanwhile, the selfsupporting structure was obtained by covalently crosslinking the ink hydrogel and removing the non-covalent support hydrogel. This system supported the patterning of multiple inks, cells, and channels in 3D space.

A tough self-healing hydrogel was synthesized as cell stimulators and implantable bioelectronics (Han et al., 2017). In 
the study, graphene oxide was partially converted to conductive graphene through polydopamine reduction, and acrylamide monomers were polymerized in situ to form the hydrogel by interactions between graphene oxide, polydopamine, and polyacrylamide. Meanwhile, the free catechol groups on polydopamine imparted self-healing property and tissue adhesion to the hydrogel via various non-covalent interactions. The hydrogel could be used not only as an adhesive electrode or motion sensor but also as an in vitro cell stimulator and in vivo implantable intramuscular electrode. For example, the hydrogel electrodes were implanted into the rabbit dorsal muscle and connected to a signal detector using the transcutaneous wires. The electrodes could record the electromyographic signal when the rabbit was interfered with external stimulation.

\section{CONCLUSIONS}

Self-healing hydrogels can be classified as robust and soft hydrogels according to mechanical properties in biomedical applications. Robust self-healing hydrogels are used as soft robots (such as implantable or wearable biosensors) with extended lifetime and mechanical performance due to repairing of the damages or fatigues. Soft self-healing hydrogels with shear-thinning properties are used in cell/drug delivery and $3 \mathrm{D}$ printing due to injection through narrow needles and retention at target sites. To facilitate biomedical applications in the future, self-healing hydrogels need to address several major concerns including (1) designing self-healing hydrogels with good biocompatibility and appropriate mechanical properties;

\section{REFERENCES}

Appel, E. A., Loh, X. J., Jones, S. T., Biedermann, F., Dreiss, C. A., and Scherman, O. A. (2012). Ultrahigh-water-content supramolecular hydrogels exhibiting multistimuli responsiveness. J. Am. Chem. Soc. 134, 11767-11773. doi: $10.1021 /$ ja3044568

Bai, T., Liu, S., Sun, F., Sinclair, A., Zhang, L., Shao, Q., et al. (2014). Zwitterionic fusion in hydrogels and spontaneous and time-independent self-healing under physiological conditions. Biomaterials 35, 3926-3933. doi: 10.1016/j.biomaterials.2014.01.077

Banerjee, S. L., and Singha, N. K. (2017). A new class of dual responsive selfhealable hydrogels based on a core crosslinked ionic block copolymer micelle prepared via RAFT polymerization and Diels-Alder "click" chemistry. Soft Matter 13, 9024-9035. doi: 10.1039/c7sm01906h

Barcan, G. A., Zhang, X., and Waymouth, R. M. (2015). Structurally dynamic hydrogels derived from 1,2-dithiolanes. J. Am. Chem. Soc. 137, 5650-5653. doi: $10.1021 /$ jacs.5b02161

Bastings, M. M., Koudstaal, S., Kieltyka, R. E., Nakano, Y., Pape, A. C., Feyen, D. A., et al. (2014). A fast $\mathrm{pH}$-switchable and self-healing supramolecular hydrogel carrier for guided, local catheter injection in the infarcted myocardium. $A d v$. Healthc. Mater. 3, 70-78. doi: 10.1002/adhm.201300076

Bergman, S. D., and Wudl, F. (2008). Mendable polymers. J. Mater. Chem. 18, 41-62. doi: 10.1039/b713953p

Canadell, J., Goossens, H., and Klumperman, B. (2011). Self-healing materials based on disulfide links. Macromolecules 44, 2536-2541. doi: $10.1021 / \mathrm{ma} 2001492$

Casuso, P., Odriozola, I., Pérez-San, A., Loinaz, I., Cabañero G., Grande, H. J., et al. (2015). Injectable and self-healing dynamic hydrogels based on
(2) better characterizing the self-healing properties with various assessment tools (such as rheological measurement, mechanical analysis, or other novel tools); (3) developing theories on selfhealing mechanisms and properties (such as chemistry, kinetics, and thermodynamics); and (4) translation by animal experiments and clinical trials. Moreover, because the self-healing properties of hydrogels are mostly determined in non-physiological environments, it would be challenging to verify that the known self-healing properties are well-maintained in physiological conditions such as with electrolytes, under mechanical stress, and in the presence of material-cell interaction. In addition, controllable biodegradability is important in selfhealing hydrogels for tissue engineering and drug delivery. In comparison with permanent crosslinks, reversible crosslinks are broken easily to facilitate biodegradation, while reversible crosslinks recover the macro- and micro-scaled damages to restrain biodegradation. Reversible equilibriums of self-healing hydrogels should be controlled according to the various applications, such as long-term drug release and cell-adaptable materials.

\section{AUTHOR CONTRIBUTIONS}

All authors listed have made a substantial, direct and intellectual contribution to the work, and approved it for publication.

\section{ACKNOWLEDGMENTS}

The work was funded by the Higher Education Sprout Project of National Taiwan University (NTU-CC-107L891101).

metal(I)-thiolate/disulfide exchange as biomaterials with tunable mechanical properties. Biomacromolecules 16, 3552-3561. doi: 10.1021/acs.biomac. 5 b00980

Casuso, P., Perez-San Vicente, A., Iribar, H., Gutierrez-Rivera, A., Izeta, A., Loinaz, I., et al. (2014). Aurophilically cross-linked "dynamic" hydrogels mimicking healthy synovial fluid properties. Chem. Commun. 50, 15199-15201. doi: $10.1039 / \mathrm{c} 4 \mathrm{cc} 05735 \mathrm{j}$

Chirila, T. V., Lee, H. H., Oddon, M., Nieuwenhuizen, M. M. L., Blakey, I., and Nicholson, T. M. (2014). Hydrogen-bonded supramolecular polymers as self-healing hydrogels: effect of a bulky adamantyl substituent in the ureidopyrimidinone monomer. J. Appl. Polym. Sci. 131:39932. doi: 10.1002/app. 39932

Cui, J., and del Campo, A. (2012). Multivalent H-bonds for self-healing hydrogels. Chem. Commun. 48, 9302-9304. doi: 10.1039/c2cc34701f

Cui, J. X., Wang, D. P., Koynov, K., and del Campo, A. (2013). 2-Ureido4-pyrimidone-based hydrogels with multiple responses. Chemphyschem 14, 2932-2938. doi: 10.1002/cphc.201300367

Dankers, P. Y., Hermans, T. M., Baughman, T. W., Kamikawa, Y., Kieltyka, R. E., Bastings, M. M., et al. (2012). Hierarchical formation of supramolecular transient networks in water: a modular injectable delivery system. Adv. Mater. 24, 2703-2709. doi: 10.1002/adma.201104072

Darabi, M. A., Khosrozadeh, A., Mbeleck, R., Liu, Y., Chang, Q., Jiang, J., et al. (2017). Skin-inspired multifunctional autonomic-intrinsic conductive selfhealing hydrogels with pressure sensitivity, stretchability, and 3D printability. Adv. Mater. 29:1700533. doi: 10.1002/adma.201700533

Deng, G., Tang, C., Li, F., Jiang, H., and Chen, Y. (2010). Covalent cross-linked polymer gels with reversible sol-gel transition and self-healing properties. Macromolecules 43, 1191-1194. doi: 10.1021/ma9022197 
Deng, G. H., Li, F. Y., Yu, H. X., Liu, F. Y., Liu, C. Y., Sun, W. X., et al. (2012). Dynamic hydrogels with an environmental adaptive self-healing ability and dual responsive sol-gel transitions. ACS Macro Lett. 1, 275-279. doi: $10.1021 / \mathrm{mz} 200195 \mathrm{n}$

Dong, R., Zhao, X., Guo, B., and Ma, P. X. (2016). Self-healing conductive injectable hydrogels with antibacterial activity as cell delivery carrier for cardiac cell therapy. ACS Appl. Mater. Interfaces 8, 17138-17150. doi: 10.1021/acsami.6b04911

Gaffey, A. C., Chen, M. H., Venkataraman, C. M., Trubelja, A., Rodell, C. B., Dinh, P. V., et al. (2015). Injectable shear-thinning hydrogels used to deliver endothelial progenitor cells, enhance cell engraftment, and improve ischemic myocardium. J. Thorac. Cardiovasc. Surg. 150, 1268-1276. doi: 10.1016/j.jtcvs.2015.07.035

Gaharwar, A. K., Avery, R. K., Assmann, A., Paul, A., McKinley, G. H., Khademhosseini, A., et al. (2014). Shear-thinning nanocomposite hydrogels for the treatment of hemorrhage. ACS Nano 8, 9833-9842. doi: 10.1021/nn503719n

Gantar, A., Drnovšek, N., Casuso, P., Pérez-San Vicente, A., Rodriguez, J., Dupin, D., et al. (2016). Injectable and self-healing dynamic hydrogel containing bioactive glass nanoparticles as a potential biomaterial for bone regeneration. RSC Adv. 6, 69156-69166. doi: 10.1039/c6ra17327f

Ghanian, M. H., Mirzadeh, H., and Baharvand, H. (2018). In situ forming, cytocompatible, and self-recoverable tough hydrogels based on dual ionic and click cross-linked alginate. Biomacromolecules 19, 1646-1662. doi: 10.1021/acs.biomac.8b00140

Grover, G. N., Lam, J., Nguyen, T. H., Segura, T., and Maynard, H. D. (2012). Biocompatible hydrogels by oxime click chemistry. Biomacromolecules 13, 3013-3017. doi: 10.1021/bm301346e

Gulyuz, U., and Okay, O. (2015). Self-healing poly(acrylic acid) hydrogels: effect of surfactant. Macromol. Symp. 358, 232-238. doi: 10.1002/masy.201500063

Han, L., Lu, X., Wang, M., Gan, D., Deng, W., Wang, K., et al. (2017). A mussel-inspired conductive, self-adhesive, and self-healable tough hydrogel as cell stimulators and implantable bioelectronics. Small 13:1601916. doi: $10.1002 /$ smll.201601916

Han, L., Zhang, Y. N., Lu, X., Wang, K. F., Wang, Z., and Zhang, H. (2016). Polydopamine nanoparticles modulating stimuli-responsive PNIPAM hydrogels with cell/tissue adhesiveness. ACS Appl. Mater. Interfaces 8, 29088-29100. doi: 10.1021/acsami.6b11043

Hao, X., Liu, H., Xie, Y. J., Fang, C., and Yang, H. Y. (2013). Thermal-responsive self-healing hydrogel based on hydrophobically modified chitosan and vesicle. Colloid Polym. Sci. 291, 1749-1758. doi: 10.1007/s00396-013-2910-4

Harada, A., Takashima, Y., and Nakahata, M. (2014). Supramolecular polymeric materials via cyclodextrin-guest interactions. Acc. Chem. Res. 47, 2128-2140. doi: $10.1021 /$ ar500109h

He, L., Fullenkamp, D. E., Rivera, J. G., and Messersmith, P. B. (2011). pH responsive self-healing hydrogels formed by boronate-catechol complexation. Chem. Commun. 47, 7497-7499. doi: 10.1039/c1cc11928a

He, L., Szopinski, D., Wu, Y., Luinstra, G. A., and Theato, P. (2015). Toward selfhealing hydrogels using one-pot thiol-ene click and borax-diol chemistry. ACS Macro Lett. 4, 673-678. doi: 10.1021/acsmacrolett.5b00336

Highley, C. B., Rodell, C. B., and Burdick, J. A. (2015). Direct 3D printing of shearthinning hydrogels into self-healing hydrogels. Adv. Mater. 27, 5075-5079. doi: 10.1002/adma.201501234

Hong, S. H., Kim, S., Park, J. P., Shin, M., Kim, K., Ryu, J. H., et al. (2018). Dynamic bonds between boronic acid and alginate: hydrogels with stretchable, self-healing, stimuli-responsive, remoldable, and adhesive properties. Biomacromolecules 19, 2053-2061. doi: 10.1021/acs.biomac. 8 b00144

Hou, S., Wang, X., Park, S., Jin, X., and Ma, P. X. (2015). Rapid self-integrating, injectable hydrogel for tissue complex regeneration. Adv. Healthc. Mater. 4, 1491-1495, 1423. doi: 10.1002/adhm.201500093

Hsieh, F.-Y., Tao, L., Wei, Y., and Hsu, S. H. (2017). A novel biodegradable selfhealing hydrogel to induce blood capillary formation. NPG Asia Mater. 9:e363. doi: $10.1038 / \mathrm{am} .2017 .23$

Hsieh, F. Y., Han, H. W., Chen, X. R., Yang, C. S., Wei, Y., and Hsu, S. H. (2018). Non-viral delivery of an optogenetic tool into cells with selfhealing hydrogel. Biomaterials 174, 31-40. doi: 10.1016/j.biomaterials.2018. 05.014
Huang, J., Deng, Y., Ren, J., Chen, G., Wang, G., Wang, F., et al. (2018). Novel in situ forming hydrogel based on xanthan and chitosan regelifying in liquids for local drug delivery. Carbohydr. Polym. 186, 54-63. doi: 10.1016/j.carbpol.2018.01.025

Huang, Y., Lawrence, P. G., and Lapitsky, Y. (2014). Self-assembly of stiff, adhesive and self-healing gels from common polyelectrolytes. Langmuir 30, 7771-7777. doi: 10.1021/la404606y

Huebsch, N., Kearney, C. J., Zhao, X., Kim, J., Cezar, C. A., Suo, Z., et al. (2014). Ultrasound-triggered disruption and self-healing of reversibly cross-linked hydrogels for drug delivery and enhanced chemotherapy. Proc. Natl. Acad. Sci. U.S.A. 111, 9762-9767. doi: 10.1073/pnas.1405469111

Ihsan, A. B., Sun, T. L., Kuroda, S., Haque, M. A., Kurokawa, T., Nakajima, T., et al. (2013). A phase diagram of neutral polyampholyte - from solution to tough hydrogel. J. Mater. Chem. B 1, 4555-4562. doi: 10.1039/c3tb20790k

Kakuta, T., Takashima, Y., Nakahata, M., Otsubo, M., Yamaguchi, H., and Harada, A. (2013). Preorganized hydrogel: self-healing properties of supramolecular hydrogels formed by polymerization of host-guest-monomers that contain cyclodextrins and hydrophobic guest groups. Adv. Mater. 25, 2849-2853. doi: 10.1002/adma.201205321

Karimi, A. R., and Khodadadi, A. (2016). Mechanically robust 3D nanostructure chitosan-based hydrogels with autonomic self-healing properties. ACS Appl. Mater. Interfaces 8, 27254-27263. doi: 10.1021/acsami.6b10375

Krogsgaard, M., Andersen, A., and Birkedal, H. (2014a). Gels and threads: musselinspired one-pot route to advanced responsive materials. Chem. Commun. 50, 13278-13281. doi: 10.1039/c4cc05293e

Krogsgaard, M., Behrens, M. A., Pedersen, J. S., and Birkedal, H. (2013). Selfhealing mussel-inspired multi-pH-responsive hydrogels. Biomacromolecules 14, 297-301. doi: 10.1021/bm301844u

Krogsgaard, M., Hansen, M. R., and Birkedal, H. (2014b). Metals \& polymers in the mix: fine-tuning the mechanical properties \& color of self-healing musselinspired hydrogels. J. Mater. Chem. B 2, 8292-8297. doi: 10.1039/c4tb01503g

Lee, H., Dellatore, S. M., Miller, W. M., and Messersmith, P. B. (2007). Musselinspired surface chemistry for multifunctional coatings. Science 318, 426-430. doi: 10.1126/science.1147241

Li, J., Su, Z. L., Ma, X. D., Xu, H. J., Shi, Z. X., Yin, J., et al. (2017). In situ polymerization induced supramolecular hydrogels of chitosan and poly(acrylic acid-acrylamide) with high toughness. Mater. Chem. Front. 1, 310-318. doi: $10.1039 / \mathrm{c} 6 q \mathrm{qm} 00002 \mathrm{a}$

Li, L., Yan, B., Yang, J., Chen, L., and Zeng, H. (2015). Novel mussel-inspired injectable self-healing hydrogel with anti-biofouling property. Adv. Mater. 27, 1294-1299. doi: 10.1002/adma.201 405166

Li, Q., Barret, D. G., Messersmith, P. B., and Holten-Andersen, N. (2016). Controlling hydrogel mechanics via bio-inspired polymer-nanoparticle bond dynamics. ACS Nano 10, 1317-1324. doi: 10.1021/acsnano.5b06692

Li, S., Wang, L., Yu, X., Wang, C., and Wang, Z. (2018a). Synthesis and characterization of a novel double cross-linked hydrogel based on Diels-Alder click reaction and coordination bonding. Mater. Sci. Eng. C Mater. Biol. Appl. 82, 299-309. doi: 10.1016/j.msec.2017.08.031

Li, S., Yi, J., Yu, X., Shi, H., Zhu, J., and Wang, L. (2018b). Preparation and characterization of acid resistant double cross-linked hydrogel for potential biomedical applications. ACS Biomater. Sci. Eng. 4, 872-883. doi: $10.1021 /$ acsbiomaterials.7b00818

Li, Y., Wang, X., Fu, Y., Wei, Y., Zhao, L., and Tao, L. (2018). A self-adapting hydrogel to improve the therapeutic effect in wound-healing. ACS Appl. Mater. Interfaces 10, 26046-26055. doi: 10.1021/acsami.8b08874

Li, Y., Zhang, Y. L., Wei, Y. N., and Tao, L. (2017). Preparation of chitosanbased injectable hydrogels and its application in 3D cell culture. J. Vis. Exp. 127:e56253. doi: 10.3791/56253

Lin, F., Yu, J., Tang, W., Zheng, J., Defante, A., Guo, K., et al. (2013). Peptidefunctionalized oxime hydrogels with tunable mechanical properties and gelation behavior. Biomacromolecules 14, 3749-3758. doi: 10.1021/bm401133r

Liu, B., Wang, Y., Miao, Y., Zhang, X., Fan, Z., Singh, G., et al. (2018). Hydrogen bonds autonomously powered gelatin methacrylate hydrogels with super-elasticity, self-heal and underwater self-adhesion for sutureless skin and stomach surgery and E-skin. Biomaterials 171, 83-96. doi: 10.1016/j.biomaterials.2018.04.023 
Liu, Q., Zhan, C., Barhoumi, A., Wang, W., Santamaria, C., McAlvin, J. B., et al. (2016). A supramolecular shear-thinning anti-inflammatory steroid hydrogel. Adv. Mater. 28, 6680-6686. doi: 10.1002/adma.20 1601147

Liu, S. L., Kang, M. M., Li, K. W., Yao, F., Oderinde, O., Fu, G. D., et al. (2018). Polysaccharide-templated preparation of mechanically-tough, conductive and self-healing hydrogels. Chem. Eng. J. 334, 2222-2230. doi: 10.1016/j.cej.2017.11.103

Liu, Y., Li, Z., Niu, N., Zou, J., and Liu, F. (2018). A simple coordination strategy for preparing a complex hydrophobic association hydrogel. J. Appl. Polym. Sci. 135:46400. doi: 10.1002/app.46400

Liu, Y. L., and Chuo, T. W. (2013). Self-healing polymers based on thermally reversible Diels-Alder chemistry. Polym. Chem. 4, 2194-2205. doi: 10.1039/c2py20957h

Loebel, C., Rodell, C. B., Chen, M. H., and Burdick, J. A. (2017). Shear-thinning and self-healing hydrogels as injectable therapeutics and for 3D-printing. Nat. Protoc. 12, 1521-1541. doi: 10.1038/nprot.2017.053

Lü, S., Gao, C., Xu, X., Bai, X., Duan, H., Gao, N., et al. (2015). Injectable and selfhealing carbohydrate-based hydrogel for cell encapsulation. ACS Appl. Mater. Interfaces 7, 13029-13037. doi: 10.1021/acsami.5b03143

Luo, F., Sun, T. L., Nakajima, T., Kurokawa, T., Zhao, Y., Sato, K., et al. (2015). Oppositely charged polyelectrolytes form tough, self-healing, and rebuildable hydrogels. Adv. Mater. 27, 2722-2727. doi: 10.1002/adma.201500140

McKee, J. R., Appel, E. A., Seitsonen, J., Kontturi, E., Scherman, O. A., and Ikkala, O. (2014). Healable, stable and stiff hydrogels: combining conflicting properties using dynamic and selective three-component recognition with reinforcing cellulose nanorods. Adv. Funct. Mater. 24, 2706-2713. doi: 10.1002/adfm.201303699

Miyamae, K., Nakahata, M., Takashima, Y., and Harada, A. (2015). Self-healing, expansion-contraction, and shape-memory properties of a preorganized supramolecular hydrogel through host-guest interactions. Angew. Chem. Int. Ed. 54, 8984-8987. doi: 10.1002/anie.201502957

Mukherjee, S., Hill, M. R., and Sumerlin, B. S. (2015). Self-healing hydrogels containing reversible oxime crosslinks. Soft Matter 11, 6152-6161. doi: $10.1039 / \mathrm{c} 5 \mathrm{sm} 00865 \mathrm{~d}$

Murphy, E. B., and Wudl, F. (2010). The world of smart healable materials. Prog. Polym. Sci. 35, 223-251. doi: 10.1016/j.progpolymsci.2009.10.006

Nakahata, M., Takashima, Y., Yamaguchi, H., and Harada, A. (2011). Redoxresponsive self-healing materials formed from host-guest polymers. Nat. Commun. 2:511. doi: 10.1038/ncomms1521

Owusu-Nkwantabisah, S., Gillmor, J. R., Switalski, S. C., and Slater, G. L. (2017). An autonomous self-healing hydrogel based on surfactant-free hydrophobic association. J. Appl. Polym. Sci. 134:44800. doi: 10.1002/app.44800

Pérez-San Vicente, A., Peroglio, M., Ernst, M., Casuso, P., Loinaz, I., Grande, H. J., et al. (2017). Self-healing dynamic hydrogel as injectable shockabsorbing artificial nucleus pulposus. Biomacromolecules 18, 2360-2370. doi: 10.1021/acs.biomac.7b00566

Qu, J., Zhao, X., Ma, P. X., and Guo, B. (2017). pH-responsive selfhealing injectable hydrogel based on N-carboxyethyl chitosan for hepatocellular carcinoma therapy. Acta Biomater. 58, 168-180. doi: 10.1016/j.actbio.2017.06.001

Rao, Z., Inoue, M., Matsuda, M., and Taguchi, T. (2011). Quick self-healing and thermo-reversible liposome gel. Colloids Surf. B Biointerfaces 82, 196-202. doi: 10.1016/j.colsurfb.2010.08.038

Ren, Y., Lou, R., Liu, X., Gao, M., Zheng, H. Z., Yang, T., et al. (2016). A self-healing hydrogel formation strategy via exploiting endothermic interactions between polyelectrolytes. Chem. Commun. 52, 6273-6276. doi: 10.1039/c6cc02472f

Rodell, C. B., Kaminski, A. L., and Burdick, J. A. (2013). Rational design of network properties in guest-host assembled and shear-thinning hyaluronic acid hydrogels. Biomacromolecules 14, 4125-4134. doi: 10.1021/bm $401280 \mathrm{z}$

Rodell, C. B., MacArthur, J. W., Dorsey, S. M., Wade, R. J., Wang, L. L., Woo, Y. J., et al. (2015a). Shear-thinning supramolecular hydrogels with secondary autonomous covalent crosslinking to modulate viscoelastic properties in vivo. Adv. Funct. Mater. 25, 636-644. doi: 10.1002/adfm.201403550

Rodell, C. B., Rai, R., Faubel, S., Burdick, J. A., and Soranno, D. E. (2015b). Local immunotherapy via delivery of interleukin-10 and transforming growth factor beta antagonist for treatment of chronic kidney disease. J. Control. Release 206, 131-139. doi: 10.1016/j.jconrel.2015.03.025

Shao, C. Y., Wang, M., Chang, H. L., Xu, F., and Yang, J. (2017). A selfhealing cellulose nanocrystal-poly(ethylene glycol) nanocomposite hydrogel via Diels-Alder click reaction. ACS Sustainable Chem. Eng. 5, 6167-6174. doi: 10.1021/acssuschemeng.7b01060

Shi, Y., Wang, M., Ma, C., Wang, Y., Li, X., and Yu, G., (2015). A conductive self-healing hybrid gel enabled by metal-ligand supramolecule and nanostructured conductive polymer. Nano Lett. 15, 6276-6281. doi: 10.1021/acs.nanolett.5b03069

Shin, M., and Lee, H. (2017). Gallol-rich hyaluronic acid hydrogels: shear-thinning, protein accumulation against concentration gradients, and degradation-resistant properties. Chem. Mater. 29, 8211-8220. doi: 10.1021/acs.chemmater.7b02267

Sileika, T. S., Barrett, D. G., Zhang, R., Lau, K. H., and Messersmith, P. B. (2013). Colorless multifunctional coatings inspired by polyphenols found in tea, chocolate, and wine. Angew. Chem. Int. Ed. 52, 10766-10770. doi: 10.1002/anie.201304922

Sun, T. L., Kurokawa, T., Kuroda, S., Ihsan, A. B., Akasaki, T., Sato, K., et al. (2013). Physical hydrogels composed of polyampholytes demonstrate high toughness and viscoelasticity. Nat. Mater. 12, 932-937. doi: 10.1038/nmat3713

Syrett, J. A., Becer, C. R., and Haddleton, D. M. (2010). Self-healing and selfmendable polymers. Polym. Chem. 1, 978-987. doi: 10.1039/c0py00104j

Toohey, K. S., Sottos, N. R., Lewis, J. A., Moore, J. S., and White, S. R. (2007). Self-healing materials with microvascular networks. Nat. Mater. 6, 581-585. doi: $10.1038 /$ nmat 1934

Tseng, T. C., Hsieh, F. Y., Theato, P., Wei, Y., and Hsu, S. H. (2017). Glucosesensitive self-healing hydrogel as sacrificial materials to fabricate vascularized constructs. Biomaterials 133, 20-28. doi: 10.1016/j.biomaterials.2017. 04.008

Tseng, T. C., Tao, L., Hsieh, F. Y., Wei, Y., Chiu, I. M., and Hsu, S. H. (2015). An injectable, self-Healing hydrogel to repair the central nervous system. $A d v$ Mater. 27, 3518-3524. doi: 10.1002/adma.201500762

Tuncaboylu, D. C., Argun, A., Sahin, M., Sari, M., and Okay, O. (2012a). Structure optimization of self-healing hydrogels formed via hydrophobic interactions. Polymer (Guildf). 53, 5513-5522. doi: 10.1016/j.polymer.2012.10.015

Tuncaboylu, D. C., Sahin, M., Argun, A., Oppermann, W., and Okay, O. (2012b). Dynamics and large strain behavior of self-healing hydrogels with and without surfactants. Macromolecules 45, 1991-2000. doi: 10.1021/ma202672y

Tuncaboylu, D. C., Sari, M., Oppermann, W., and Okay, O. (2011). Tough and self-healing hydrogels formed via hydrophobic interactions. Macromolecules 44, 4997-5005. doi: 10.1021/ma200579v

Wang, H., and Heilshorn, S. C. (2015). Adaptable hydrogel networks with reversible linkages for tissue engineering. Adv. Mater. 27, 3717-3736. doi: 10.1002/adma.201501558

Wang, J. Y., Wang, D., Yan, H., Tao, L., Wei, Y., Li, Y. S., et al. (2017). An injectable ionic hydrogel inducing high temperature hyperthermia for microwave tumor ablation. J. Mater. Chem. B 5, 4110-4120. doi: 10.1039/c7tb00556c

Wang, L. L., Highley, C. B., Yeh, Y. C., Galarraga, J. H., Uman, S., and Burdick, J. A. (2018). Three-dimensional extrusion bioprinting of single- and doublenetwork hydrogels containing dynamic covalent crosslinks. J. Biomed. Mater. Res. A 106, 865-875. doi: 10.1002/jbm.a.36323

Wang, L. L., Sloand, J. N., Gaffey, A. C., Venkataraman, C. M., Wang, Z. C., Trubelja, A., et al. (2017). Injectable, guest-host assembled polyethylenimine hydrogel for siRNA delivery. Biomacromolecules 18, 77-86. doi: 10.1021/acs.biomac.6b01378

Wang, Y., Park, J. P., Hong, S. H., and Lee, H. (2016). Biologically inspired materials exhibiting repeatable regeneration with self-sealing capabilities without external stimuli or catalysts. Adv. Mater. 28, 9961-9968. doi: 10.1002/adma.201603290

Wei, H., Du, S., Liu, Y., Zhao, H., Chen, C., Li, Z., et al. (2014). Tunable, luminescent, and self-healing hybrid hydrogels of polyoxometalates and triblock copolymers based on electrostatic assembly. Chem. Commun. 50, 1447-1450. doi: 10.1039/c3cc48732f

Wei, Z., He, J., Liang, T., Oh, H., Athas, J., Tong, Z., et al. (2013). Autonomous self-healing of poly(acrylic acid) hydrogels induced by the migration of ferric ions. Polym. Chem. 4, 4601-4605. doi: 10.1039/c3py00692a 
Wei, Z., Yang, J. H., Liu, Z. Q., Xu, F., Zhou, J. X., Zrinyi, M., et al. (2015). Novel biocompatible polysaccharide-based self-healing hydrogel. Adv. Funct. Mater. 25, 1352-1359. doi: 10.1002/adfm.201401502

Wei, Z., Yang, J. H., Zhou, J. X., Xu, F., Zrínyi, M., Dussault, P. H., et al. (2014). Self-healing gels based on constitutional dynamic chemistry and their potential applications. Chem. Soc. Rev. 43, 8114-8131. doi: 10.1039/c4cs00219a

White, S. R., Sottos, N. R., Geubelle, P. H., Moore, J. S., Kessler, M. R., Sriram, S. R., et al. (2001). Autonomic healing of polymer composites. Nature 409, 794-797. doi: $10.1038 / 35057232$

Xia, L. Y., Zhang, X. D., Cao, M., Chen, Z., and Wu, F. G. (2017). Enhanced fluorescence emission and singlet oxygen generation of photosensitizers embedded in injectable hydrogels for imaging guided photodynamic cancer therapy. Biomacromolecules 18, 3073-3081. doi: 10.1021/acs.biomac.7b00725

Xie, W., Gao, Q., Guo, Z., Wang, D., Gao, F., Wang, X., et al. (2017). Injectable and self-healing thermosensitive magnetic hydrogel for asynchronous control release of doxorubicin and docetaxel to treat triple-negative breast cancer. ACS Appl. Mater. Interfaces 9, 33660-33673. doi: 10.1021/acsami.7b10699

Xing, R., Liu, K., Jiao, T., Zhang, N., Ma, K., Zhang, R. Y., et al. (2016). An injectable self-assembling collagen-gold hybrid hydrogel for combinatorial antitumor photothermal/photodynamic therapy. Adv. Mater. 28, 3669-3676. doi: 10.1002/adma.201600284

Xu, G. Z., Xiao, Y., Cheng, L., Zhou, R. H., Xu, H., Chai, Y. M., et al. (2017). Synthesis and rheological investigation of self-healable deferoxamine grafted alginate hydrogel. J. Polym. Sci. Pol. Phys. 55, 856-865. doi: 10.1002/polb.24334

Yamaguchi, H., Kobayashi, Y., Kobayashi, R., Takashima, Y., Hashidzume, A., and Harada, A. (2012). Photoswitchable gel assembly based on molecular recognition. Nat. Commun. 3:603. doi: 10.1038/ncomms1617

Yang, B., Zhang, Y. L., Zhang, X. Y., Tao, L., Li, S. X., and Wei, Y. (2012). Facilely prepared inexpensive and biocompatible self-healing hydrogel: a new injectable cell therapy carrier. Polym. Chem. 3, 3235-3238. doi: 10.1039/c2py20627g

Yang, L., Li, Y. S., Gou, Y. Z., Wang, X., Zhao, X. M., and Tao, L. (2017). Improving tumor chemotherapy effect using an injectable self-healing hydrogel as drug carrier. Polym. Chem. 8, 3071-3076. doi: 10.1039/c7py00112f

Yang, W. J., Tao, X., Zhao, T., Weng, L., Kang, E.-T., and Wang, L. (2015). Antifouling and antibacterial hydrogel coatings with self-healing properties based on a dynamic disulfide exchange reaction. Polym. Chem. 6, 7027-7035. doi: $10.1039 / \mathrm{c} 5$ py00936g

Yang, X. F., Liu, G. Q., Peng, L., Guo, J. H., Tao, L., Yuan, J. Y., et al. (2017). Highly efficient self-healable and dual responsive cellulose-based hydrogels for controlled release and 3D cell culture. Adv. Funct. Mater. 27:1703174. doi: 10.1002/adfm.201703174

Yavvari, P. S., Pal, S., Kumar, S., Kar, A., Awasthi, A. K., Naaz, A., et al. (2017). Injectable, self-healing chimeric catechol-Fe(III) hydrogel for localized combination cancer therapy. ACS Biomater. Sci. Eng. 3, 3404-3413. doi: 10.1021/acsbiomaterials.7b00741

Ye, X., Li, X., Shen, Y. Q., Chang, G. J., Yang, J. X., and Gu, Z. W. (2017). Self-healing $\mathrm{pH}$-sensitive cytosine- and guanosine-modified hyaluronic acid hydrogels via hydrogen bonding. Polymer (Guildf). 108, 348-360. doi: 10.1016/j.polymer.2016.11.063

Yesilyurt, V., Webber, M. J., Appel, E. A., Godwin, C., Langer, R., and Anderson, D. G. (2016). Injectable self-healing glucose-responsive hydrogels with $\mathrm{pH}$-regulated mechanical properties. Adv. Mater. 28, 86-91. doi: 10.1002/adma.201502902

Yoon, J. A., Kamada, J., Koynov, K., Mohin, J., Nicolay,, R., Zhang, Y., et al. (2011). Self-healing polymer films based on thiol-disulfide exchange reactions and selfhealing kinetics measured using atomic force microscopy. Macromolecules 45, 142-149. doi: 10.1021/ma2015134

Yu, F., Cao, X., Du, J., Wang, G., and Chen, X. (2015). Multifunctional hydrogel with good structure integrity, self-healing, and tissue-adhesive property formed by combining Diels-Alder click reaction and acylhydrazone bond. ACS Appl. Mater. Interfaces 7, 24023-24031. doi: 10.1021/acsami.5b06896
Yu, H., Wang, Y., Yang, H., Peng, K., and Zhang, X. (2017). Injectable selfhealing hydrogels formed via thiol/disulfide exchange of thiol functionalized F127 and dithiolane modified PEG. J. Mater. Chem. B 5, 4121-4127. doi: 10.1039/c7tb00746a

Zhang, G., Ngai, T., Deng, Y., and Wang, C. (2016). An injectable hydrogel with excellent self-healing property based on quadruple hydrogen bonding. Macromol. Chem. Phys. 217, 2172-2181. doi: 10.1002/macp.201 600319

Zhang, H., Xia, H., and Zhao, Y. (2012). Poly(vinyl alcohol) hydrogel can autonomously self-heal. ACS Macro Lett. 1, 1233-1236. doi: $10.1021 / \mathrm{mz} 300451 \mathrm{r}$

Zhang, X., and Waymouth, R. M. (2017). 1,2-Dithiolane-derived dynamic, covalent materials: cooperative self-assembly and reversible cross-linking. $J$. Am. Chem. Soc. 139, 3822-3833. doi: 10.1021/jacs.7b00039

Zhang, Y., Tao, L., Li, S., and Wei, Y. (2011). Synthesis of multiresponsive and dynamic chitosan-based hydrogels for controlled release of bioactive molecules. Biomacromolecules 12, 2894-2901. doi: 10.1021/bm200423f

Zhang, Y. L., Fu, C. K., Li, Y. S., Wang, K., Wang, X., Wei, Y., et al. (2017). Synthesis of an injectable, self-healable and dual responsive hydrogel for drug delivery and 3D cell cultivation. Polym. Chem. 8, 537-544. doi: 10.1039/c6py01704e

Zhang, Z., Li, T. T., Chen, B., Wang, S., and Guo, Z. Y. (2017). Self-healing supramolecular hydrogel of poly(vinyl alcohol)/chitosan carbon dots. J. Mater. Sci. 52, 10614-10623. doi: 10.1007/s10853-017-1222-3

Zhao, J., Xu, R., Luo, G., Wu, J., and Xia, H. (2016). A self-healing, re-moldable and biocompatible crosslinked polysiloxane elastomer. J. Mater. Chem. B 4, 982-989. doi: 10.1039/c5tb02036k

Zhao, X., Wu, H., Guo, B., Dong, R., Qiu, Y., and Ma, P. X. (2017). Antibacterial anti-oxidant electroactive injectable hydrogel as self-healing wound dressing with hemostasis and adhesiveness for cutaneous wound healing. Biomaterials 122, 34-47. doi: 10.1016/j.biomaterials.2017.01.011

Zheng, Y., Hashidzume, A., and Harada, A. (2013). pH-responsive self-assembly by molecular recognition on a macroscopic scale. Macromol. Rapid Commun. 34, 1062-1066. doi: 10.1002/marc.201300324

Zheng, Y., Hashidzume, A., Takashima, Y., Yamaguchi, H., and Harada, A. (2012). Temperature-sensitive macroscopic assembly based on molecular recognition. ACS Macro Lett. 1, 1083-1085. doi: 10.1021/mz300338d

Zhu, C., Zhao, J., Kempe, K., Wilson, P., Wang, J., Velkov, T., et al. (2017). A hydrogel-based localized release of colistin for antimicrobial treatment of burn wound infection. Macromol. Biosci. 17:1600320. doi: 10.1002/mabi.201600320

Zhu, D., Wang, H., Trinh, P., Heilshorn, S. C., and Yang, F. (2017). Elastinlike protein-hyaluronic acid (ELP-HA) hydrogels with decoupled mechanical and biochemical cues for cartilage regeneration. Biomaterials 127, 132-140. doi: 10.1016/j.biomaterials.2017.02.010

Zhu, S. K., Wang, J. X., Yan, H. R., Wang, Y. Y., Zhao, Y. C., Feng, B., et al. (2017). An injectable supramolecular self-healing bio-hydrogel with high stretchability, extensibility and ductility, and a high swelling ratio. J. Mater. Chem. B 5, 7021-7034. doi: 10.1039/c7tb01183k

Zou, W., Dong, J., Luo, Y., Zhao, Q., and Xie, T. (2017). Dynamic covalent polymer networks: from old chemistry to modern day innovations. Adv. Mater. 29:1600320. doi: 10.1002/adma.201606100

Conflict of Interest Statement: The authors declare that the research was conducted in the absence of any commercial or financial relationships that could be construed as a potential conflict of interest.

Copyright (c) $2018 \mathrm{Liu}$ and Hsu. This is an open-access article distributed under the terms of the Creative Commons Attribution License (CC BY). The use, distribution or reproduction in other forums is permitted, provided the original author $(s)$ and the copyright owner(s) are credited and that the original publication in this journal is cited, in accordance with accepted academic practice. No use, distribution or reproduction is permitted which does not comply with these terms. 